\title{
Structural and Electronic Properties of Gold Contacts on CdZnTe with Different Surface Finishes for Radiation Detector...
}

Article in Journal of Electronic Materials · August 2014

DOI: $10.1007 /$ s11664-014-3167-5

CITATIONS

7

7 authors, including:

\section{Suleyman Tari}

University of Illinois at Chicago

20 PUBLICATIONS 163 CITATIONS

SEE PROFILE

\section{Jin Li}

California State University, Northridge 7 PUBLICATIONS 33 CITATIONS

SEE PROFILE
READS

63

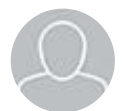

\section{Christoph H. Grein}

University of Illinois at Chicago

218 PUBLICATIONS 2,915 CITATIONS

SEE PROFILE

\section{Nicholas Kioussis}

California State University, Northridge 192 PUBLICATIONS 2,311 CITATIONS

SEE PROFILE

Some of the authors of this publication are also working on these related projects: 


\title{
Structural and Electronic Properties of Gold Contacts on CdZnTe with Different Surface Finishes for Radiation Detector Applications
}

\author{
S. TARI,${ }^{1,4}$ F. AQARIDEN,${ }^{1}$ Y. CHANG,${ }^{1}$ A. CIANI, ${ }^{1}$ C. GREIN,${ }^{2}$ JIN LI,${ }^{3}$ \\ and N. KIOUSSIS ${ }^{3}$ \\ 1.-Sivananthan Laboratories, Inc., 590 Territorial Drive, Ste H, Bolingbrook, IL 60440, USA. \\ 2.-Department of Physics, University of Illinois at Chicago, Chicago, IL 60607-7059, USA. \\ 3.-W. M. Keck Computational Materials Theory Center, California State University at Northridge, \\ Northridge, CA 91330-8268, USA. 4.—e-mail: stari@sivananthanlabs.us
}

\begin{abstract}
State-of-the-art room-temperature, high-resolution x-ray and gamma-ray semiconductor detectors can be fabricated from CdZnTe (CZT) crystals. The structural and electronic properties of the CZT surface, especially the contact interfaces, can have a substantial effect on radiation detector performance, for example leakage current, signal-to-noise ratio, and energy resolution, especially for soft x-rays and large pixilated arrays. Atomically smooth and defect-free surfaces are desirable for high-performance CZT-based detectors; chemo-mechanical polishing (CMP) is typically performed to produce such surfaces. The electrical behavior of the metal/CZT interface varies substantially with surface preparation before contact deposition, and with choice of metal and deposition technique. We report a systematic study of the structural and electronic properties of gold (Au) contacts on CZT prepared with different surface finishes. We observed subsurface damage under Au contacts on CMPfinished CZT and abrupt interfaces for Au on chemically-polished (CP) CZT. Schottky barrier formation was observed for Au contacts, irrespective of surface finish, and less charge trapping and low surface resistance were observed for CP-finished surfaces. Pre-deposition surface treatment produced interfaces free from oxide layers.
\end{abstract}

Key words: CZT, radiation detector, polishing, leakage current, XPS, metal contacts, ohmic

\section{INTRODUCTION}

$\mathrm{CdZnTe}(\mathrm{CZT})$ is a promising material for highperformance semiconductor x-ray and gamma-ray radiation detectors that can operate at room temperature. $^{1-3}$ CZT bulk crystals can contain significant concentrations of defects, for example $\mathrm{Cd}$ vacancies, Te precipitates, impurities, and grains. A comprehensive international effort is being made to improve the crystalline quality of $\mathrm{CZT} ;{ }^{4}$ however, the performance of a radiation detector depends not only on the quality of its bulk material but also on

(Received November 20, 2013; accepted April 4, 2014; published online May 5, 2014) its surfaces and contacts. Surfaces and contacts can be the dominant factors affecting detector performance for some device structures and applications; this is especially true when CZT is used for soft $\mathrm{x}$-ray detection (low signal-to-noise ratio) or in arrays with small pixel pitch (large surface-tovolume ratio and short inter-pixel distance). Roughness, oxidation, defects, or contamination can alter the electronic structure of the surface and reduce detector performance, so atomically smooth and defect-free surfaces are necessary to obtain high-performance CZT-based radiation detectors. Research addressing contact electrode improvements, with the purpose of reducing detector leakage currents, has been reported. ${ }^{5-7}$ The effects of 
different chemical solutions on the relationship between surface roughness and leakage current of CZT detectors have also been studied. , $^{8}$

$\mathrm{Au}$ is a frequent choice for the anode contact, because it can be deposited by use of several techniques and a sharp interface with little intermixing is usually obtained. ${ }^{9,10}$ A low work-function cathode, for example In, may be combined with a Au anode to create a Schottky device and improve energy resolution. ${ }^{11}$ Surface preparation may affect Schottky barrier height, carrier injection and collection, and leakage current.

Chemo-mechanical polishing (CMP) is typically used to obtain a smooth, planar surface. After the CMP process, CZT may be chemically etched in a bromine-methanol $(\mathrm{Br}-\mathrm{MeOH})$ solution to remove the fine scratches that result from the CMP process or the $\mathrm{TeO}_{2}$ layer that forms after polishing. Chemical etching substantially alters the CZT surface and introduces a variety of defects. For example, recent work ${ }^{9}$ revealed that etching with $1 \%$ $\mathrm{Br}-\mathrm{MeOH}$ removed the passivating surface oxide and increased leakage currents. Some passivating oxide between electrodes may be desirable, but an oxide layer between the CZT and electrode could interfere with carrier injection and extraction, and an excessively thick passivating layer could effectively constitute a jacket of amorphous semiconductor and increase leakage. It is necessary and nontrivial to create reproducible surfaces by use of chemical processes. The effects of polishing and etching of CZT surfaces have recently been reported. ${ }^{12}$ Chemical polishing (CP) as a final production step creates a mirror-like surface and eliminates the need to etch the CZT.

In this work we studied the structural and electronic properties of $\mathrm{Au}$ contacts deposited by electron beam (E-beam) evaporation on $\mathrm{Cd}_{0.9} \mathrm{Zn}_{0.1} \mathrm{Te}$ with a variety of surface finishes.

\section{EXPERIMENT}

We regularly achieve, by use of successive CMP and $\mathrm{CP}$ processes, $\mathrm{Cd}_{0.90} \mathrm{Zn}_{0.10} \mathrm{Te}$ crystals and $\mathrm{Cd}_{0.96} \mathrm{Zn}_{0.04} \mathrm{Te}$ wafers with root mean square (rms) surface roughness of $0.8 \mathrm{~nm}$, as determined by atomic force microscopy (AFM). State-of-the-art rms roughness is $0.5 \mathrm{~nm}$ for $\mathrm{Cd}_{0.96} \mathrm{Zn}_{0.04}$ Te wafers available from Nikko Materials. We previously reported use of $\mathrm{Cd}_{0.96} \mathrm{Zn}_{0.04} \mathrm{Te}$ wafer-polishing methods on bulk $\mathrm{Cd}_{0.9} \mathrm{Zn}_{0.1} \mathrm{Te}$ crystals for radiation detection applications. ${ }^{13}$

Single-crystal $\mathrm{Cd}_{0.9} \mathrm{Zn}_{0.1} \mathrm{Te}$ samples of size $20 \mathrm{~mm} \times$ $20 \mathrm{~mm} \times 5 \mathrm{~mm}$ were obtained from a commercial supplier. The surface morphology of the as-received samples was characterized by use of differential interference contrast (DIC) optical microscopy (OM). Some of the samples were diced into $10 \mathrm{~mm} \times 10 \mathrm{~mm} \times$ $5 \mathrm{~mm}$ pieces for experiments using planar contacts whereas others were left intact for experiments with transmission line method (TLM) contacts. The samples were CMP and CP processed. Details of the polishing process have been reported elsewhere. ${ }^{13}$ After CP, the CZT surfaces are smooth and scratch-free, and require no further processing. Immediately before contact deposition, the samples were briefly etched in an $\mathrm{HCl}$ solution to remove the oxide layer. Au contacts were deposited with either of the two contact geometries shown in Fig. 1. TLM contacts were deposited on as-received, CMP-finished, and CP-finished surfaces to investigate the effects of surface roughness. Planar contacts were deposited on CP-finished samples, only, to determine bulk resistance and Schottky barrier heights.

Structural, chemical, and electronic characterization techniques were used to study the CZT surface and the $\mathrm{Au} / \mathrm{CZT}$ interface. High-resolution scanning transmission electron microscopy (HR-STEM) and scanning electron microscopy (SEM) were used to inspect the interface structure and chemistry. The surface chemical composition was analyzed by x-ray photoelectron spectroscopy (XPS) by use of a spectrometer with a monochromatic and focused Al $K_{\alpha}$ source $(E=1486.6 \mathrm{eV})$. Survey spectra were recorded with a $1000 \mu \mathrm{m}$ spot diameter, and core-level spectra were recorded with a $600 \mu \mathrm{m}$ spot diameter and $50 \mathrm{eV}$ pass energy. The corresponding energy resolution for core-level scans is $0.9 \mathrm{eV}$, as measured by the full width at half-maximum of the $\mathrm{Au} 4 f$ core level. Current voltage $(I-V)$ measurements were made between TLM contacts on the same surface and across the CZT thickness to determine surface leakage currents and bulk resistance.

\section{RESULTS}

DIC images of the surfaces of as-received, CMPprocessed, and CP-processed CZT are shown in Fig. 2. A high density of large scratches is present on the as-received CZT, as a result of the mechanical polishing performed by the manufacturer. After CMP, the scratches become fine and less dense. After CP, the surface is smooth, with no indication of scratches or defects. CP is similar to etching in $\mathrm{Br}-\mathrm{MeOH}$ in that it removes $\mathrm{CZT}$ and oxide layers;

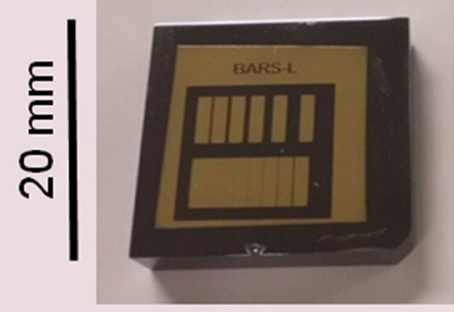

(a)

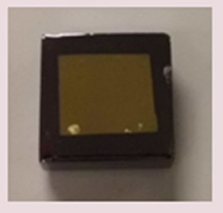

(b)
Fig. 1. Au deposited on CP-finished CZT: (a) TLM structure on either A or B face, and (b) planar contacts on opposite sides (A and B faces). 
however, our CP process preserves flatness while removing scratches and damage layers, whereas etching in $\mathrm{Br}-\mathrm{MeOH}$ tends to create surface undulations (orange peel) on a sub-millimeter scale and roughness on a sub-micron to nanometer scale. Etching the CZT surface is usually avoided, because doing so tends to increase leakage currents ${ }^{9}$ (without post-etch processing), but chemical removal of the rough surface may be important to improve device performance.

Figure 3 shows cross-sectional SEM images of the $\mathrm{Au} / \mathrm{CZT}$ interfaces on CMP and CP-finished crystals. Despite substantial reduction by CMP of the density and sizes of scratches visible with OM, SEM reveals substantial sub-surface damage that penetrates to $5 \mu \mathrm{m}$ below the Au/CZT interface. Our CP process completely removes this damage layer and produces an abrupt interface between the Au electrode and the single-crystal material.

The HR-STEM micrograph shown in Fig. 4 reveals the atomic arrangement of the $\mathrm{Au} / \mathrm{CZT}$ interface on a CP-finished crystal. The CZT is monocrystalline with clear columns of atoms whereas the $\mathrm{Au}$ is amorphous. Energy-dispersive spectroscopy (EDS) indicates that $\mathrm{Cd}$ and $\mathrm{Au}$ are largely segregated across the interface whereas Te seems to intermix with the Au layer. The depth of this intermixing is only a few nanometers and is likely to be the result of reaction of $\mathrm{Au}$ with excess Te on the Te-rich CZT surface. The interface also has undulations of a few nanometers, and the electron beam may have sampled through this undulation, effectively measuring the interface as more diffuse than it actually is.

XPS survey scans and high-resolution peak scans around the Te 3d energy were performed on CZT samples after CMP processing, $\mathrm{HCl}$ etching, deposition of a 200-nm Au contact, and thinning the $\mathrm{Au}$ contact. After etching in $\mathrm{HCl}$, the $\mathrm{CZT}$ surface is Te-rich and a native oxide layer forms slowly within a few hours when exposed to the atmosphere.

All of the expected photoelectron peaks were seen in the survey scans. Figure 5 shows the XPS spectra of Te $3 \mathrm{~d}$ peaks. The spectra are normalized to the Te $3 \mathrm{~d}_{5 / 2}$ peak for comparison purposes, and shifted in intensity to clearly reveal the differences. The positions of the Te $3 \mathrm{~d}_{5 / 2}$ and $\mathrm{Te} 3 \mathrm{~d}_{3 / 2}$ peaks on the low-energy side were found to be $572.8 \mathrm{eV}$ and $582.8 \mathrm{eV}$, respectively. All spectra contain clear Te $3 \mathrm{~d}$ peaks. After the polishing process, a low-intensity peak at $576.2 \mathrm{eV}$ is observed; this is indicative of $\mathrm{TeO}_{2}$ formation. The thickness of the oxide is estimated, on the basis of compositional peak analysis, to be approximately $\sim 1-2 \mathrm{~nm}$. The $\mathrm{TeO}_{2}$ peak is expected after polishing because the CZT surface is Te-rich and vulnerable to oxidation. There is no $\mathrm{TeO}_{2}$ peak immediately after etching CZT with $\mathrm{HCl}$. An XPS spectrum was also measured after depositing a $200 \mathrm{~nm} \mathrm{Au}$ contact; this showed Au peaks only (not shown). After removal of the Au contact, an XPS scan of the $\mathrm{Au} / \mathrm{CZT}$ interface revealed $\mathrm{Au}$ and CdTe but no, or minimal, $\mathrm{TeO}_{2}$ formation. A weak $\mathrm{TeO}_{2}$ peak appears but has about the same magnitude as the noise, so we can estimate that the
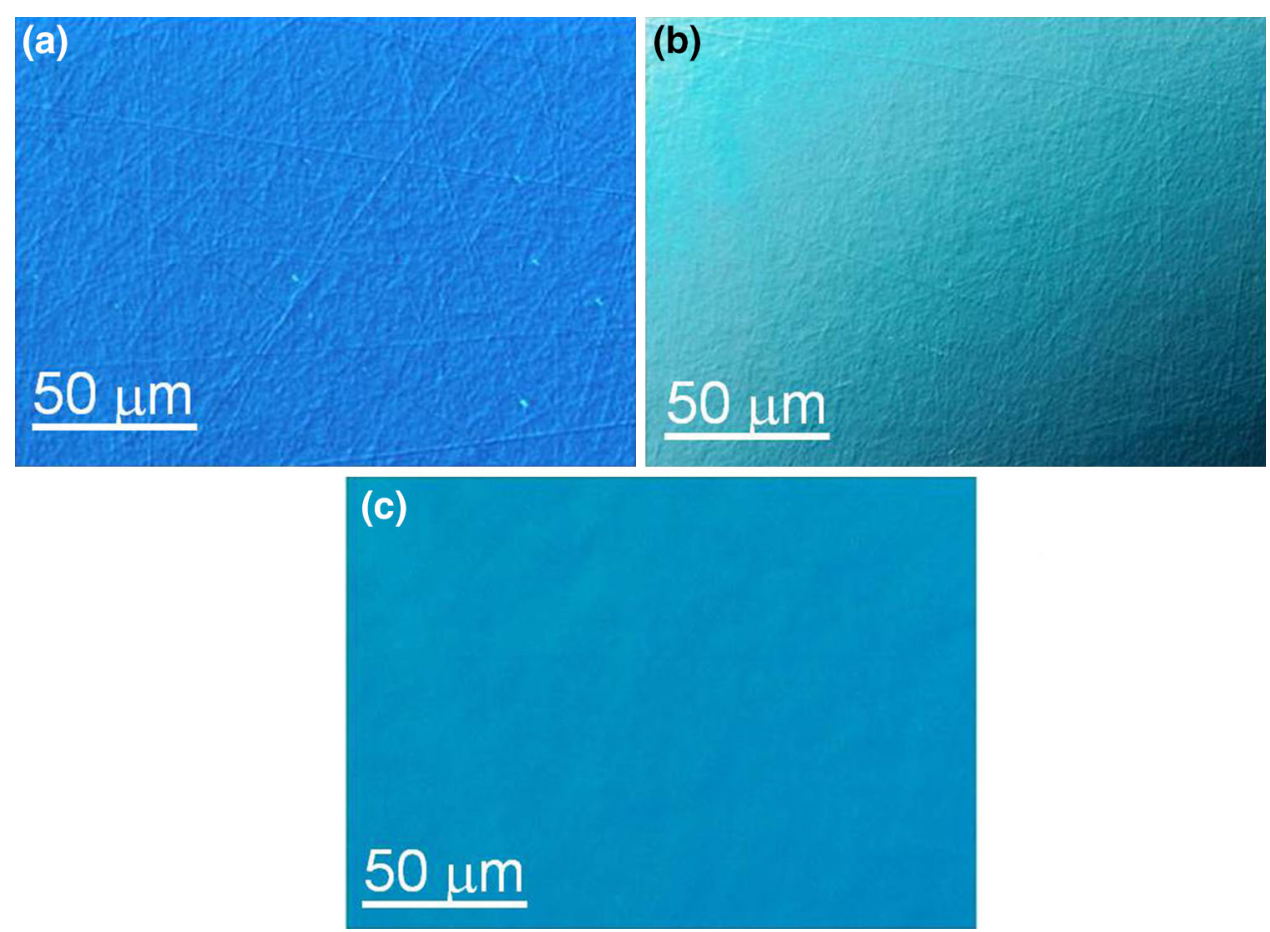

Fig. 2. OM images of CZT as received (a), and after CMP (b) and CP (c) finishes. 

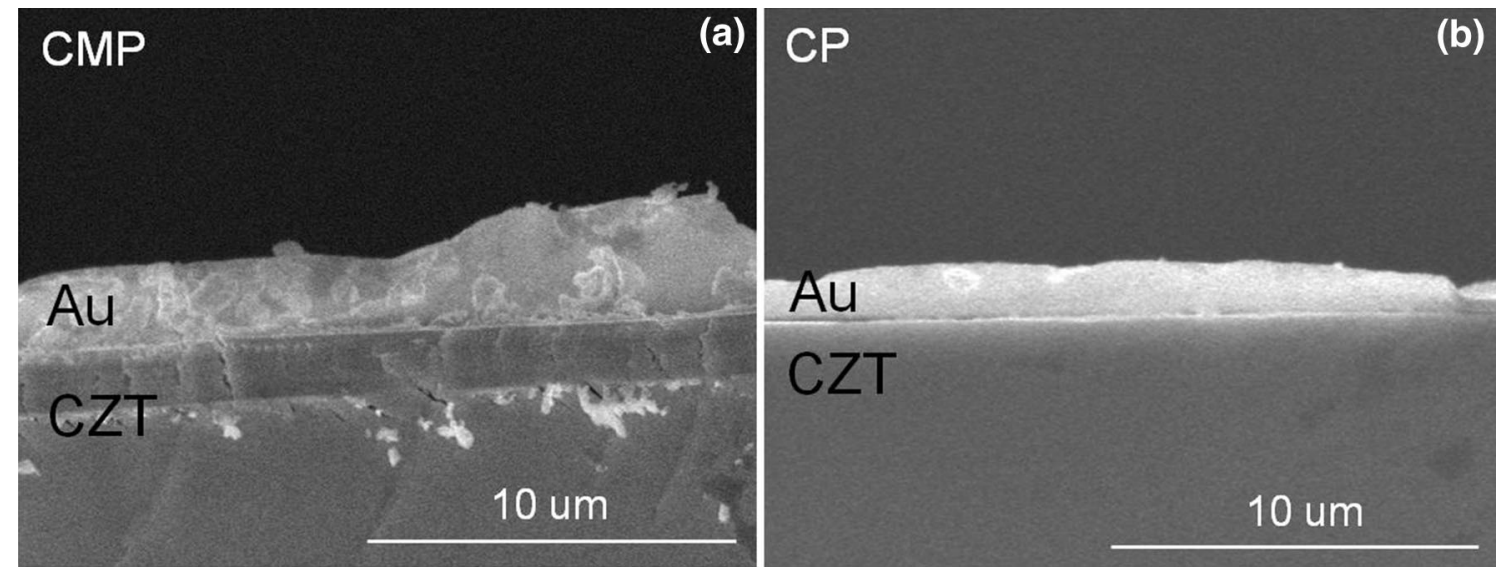

Fig. 3. SEM cross-sections of Au/CZT interfaces after (a) CMP and (b) CP finishes.

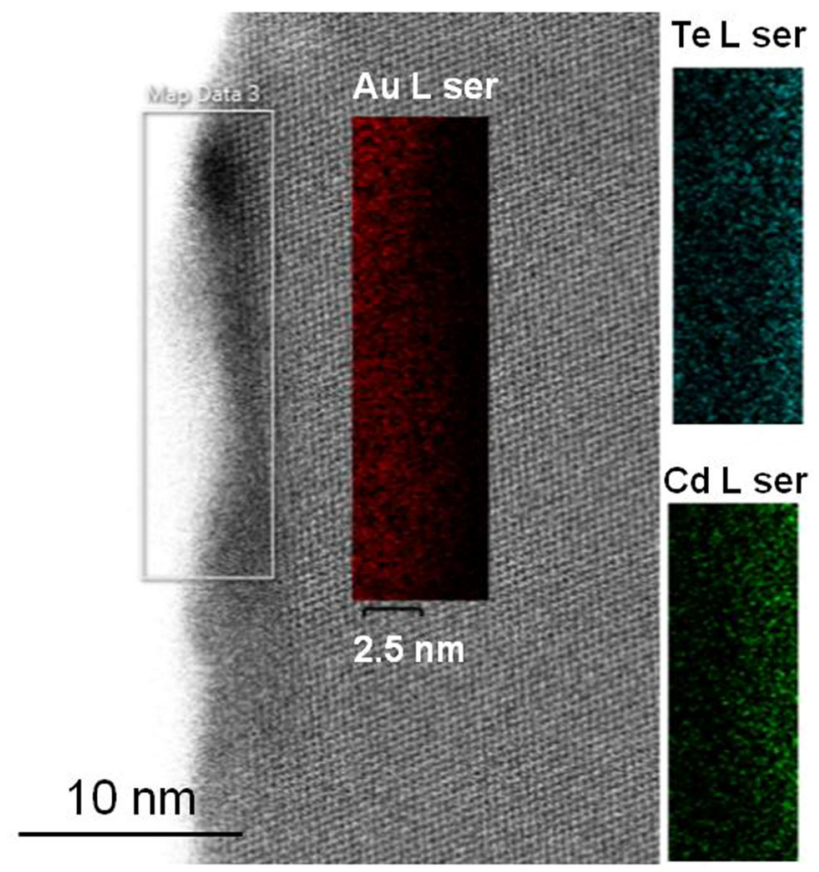

Fig. 4. HR-STEM EDS image of Au/CZT interface.

oxide layer is less than a full monolayer thick. XPS and EDS analysis confirms there is no or minimal intermixing at the interface of $\mathrm{Au} / \mathrm{CZT}$ and the interface is essentially oxide-free.

The $I-V$ characteristics of the planar and TLM structures were measured. Figure 6 shows the $I-V$ curve for the $\mathrm{Au} / \mathrm{CZT} / \mathrm{Au}$ photoconductor device with planar contacts. Its behavior is typical of that of back-to-back Schottky diodes: the forward-biased current through one junction is limited by the reverse-bias saturation current of the other. With regard to barrier height and saturation current, the quality of the surface finish had no discernible effect on the $I-V$ measurements through the bulk; however, the surface finish has an effect on hysteresis. To perform the measurements, the applied voltage was started at the maximum negative bias

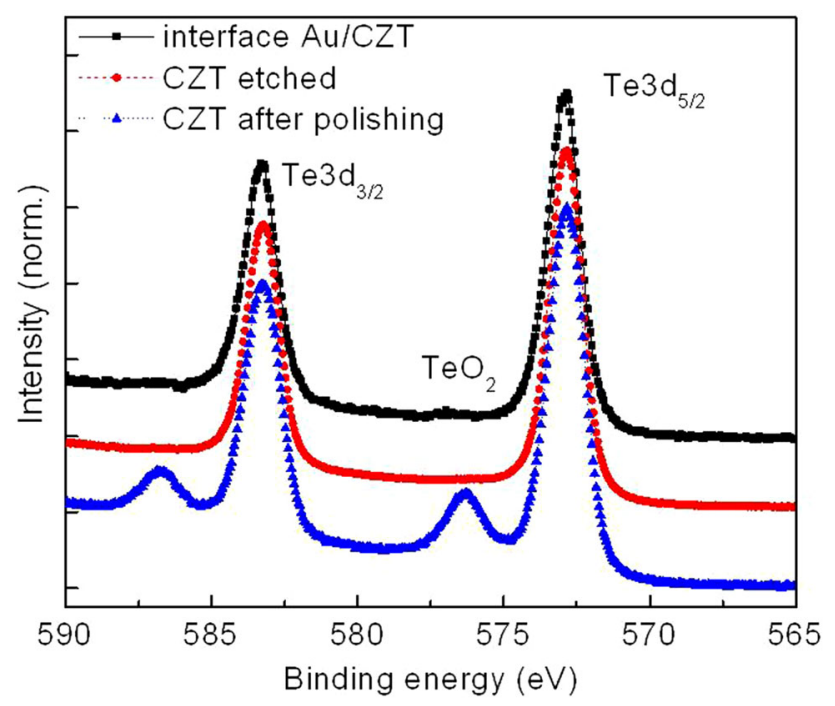

Fig. 5. XPS spectra of the Te 3d peak after $\mathrm{CP}, \mathrm{HCl}$ etching, and thinning of the Au contact (i.e. the Au/CZT interface). For clarity, the three lines are separated by one unit so that they do not overlap. Intensity is a nominal unit scaled to the $\mathrm{Te} 3 \mathrm{~d}_{5 / 2}$ peak.

$(-120 \mathrm{~V})$, stepped up to the maximum positive bias, and then stepped back down to the maximum negative bias. Under negative bias some hysteresis was observed for every sample, probably because of trapping of charges; however, hysteresis was less for CP samples and a steady current was usually reached more rapidly than for CMP or as-received samples.

The effect of polishing is clearly evident for TLM device structures, the $I-V$ curves for which are shown in Fig. 7. For contacts on as-received and CMP-polished samples, $I-V$ curves were dominated by junctions in which the bulk resistance of the CZT had little effect on the saturation current. It is also apparent that hysteresis was less for the CMP-polished sample than for as-received samples. For the CP-polished sample, clear, almost linear dependence of current on contact separation was observed, with 


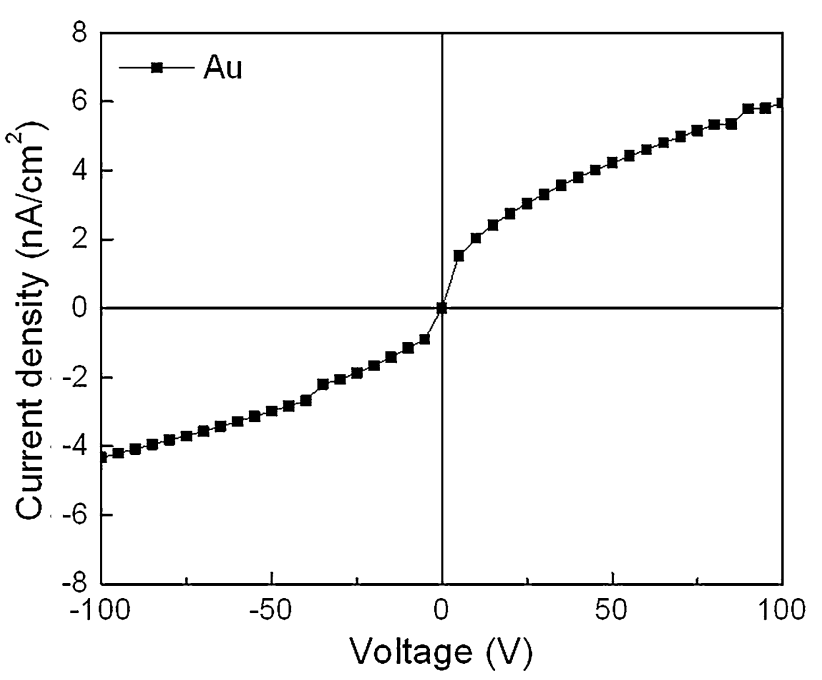

Fig. 6. $I-V$ for the Au/CZT/Au planar structure.

high surface currents bypassing the Schottky junction and very little sign of a Schottky barrier or hysteresis.

\section{SUMMARY AND CONCLUSIONS}

CZT crystals were polished by using CMP and CP processes, and Au contacts with planar or TLM geometry were deposited by E-beam evaporation. Immediately before contact deposition, the crystals were briefly etched in $\mathrm{HCl}$ solution to remove the native oxide layer. OM images revealed clear progression of surface smoothness, and SEM images showed that even the smoothest CMP polished surface still had subsurface damage extending several microns deep. The CP process produced a surface that was smooth and free of subsurface damage, and TEM imaging revealed that the $\mathrm{Au} /$ CZT interface had a few nanometers of waviness and only a nanometer-wide mixed region in which Te had penetrated into the Au. XPS analysis of the Au contact showed that very little oxide developed between the CZT and Au. Current-voltage measurements of planar devices revealed that $\mathrm{Au}$ formed a Schottky junction with CZT, irrespective of surface roughness, and the saturation current was also independent of roughness; however, the amount of hysteresis in the $I-V$ measurements suggested that interface smoothness may reduce charge trapping. Current-voltage measurements for TLM devices showed that CMP reduced hysteresis whereas CP allowed a significant amount of surface current to bypass the Schottky junction.

Previous papers have generally attributed increased leakage currents after etching to removal of the passivating oxide layer; however, the XPS results showed that a native oxide layer forms
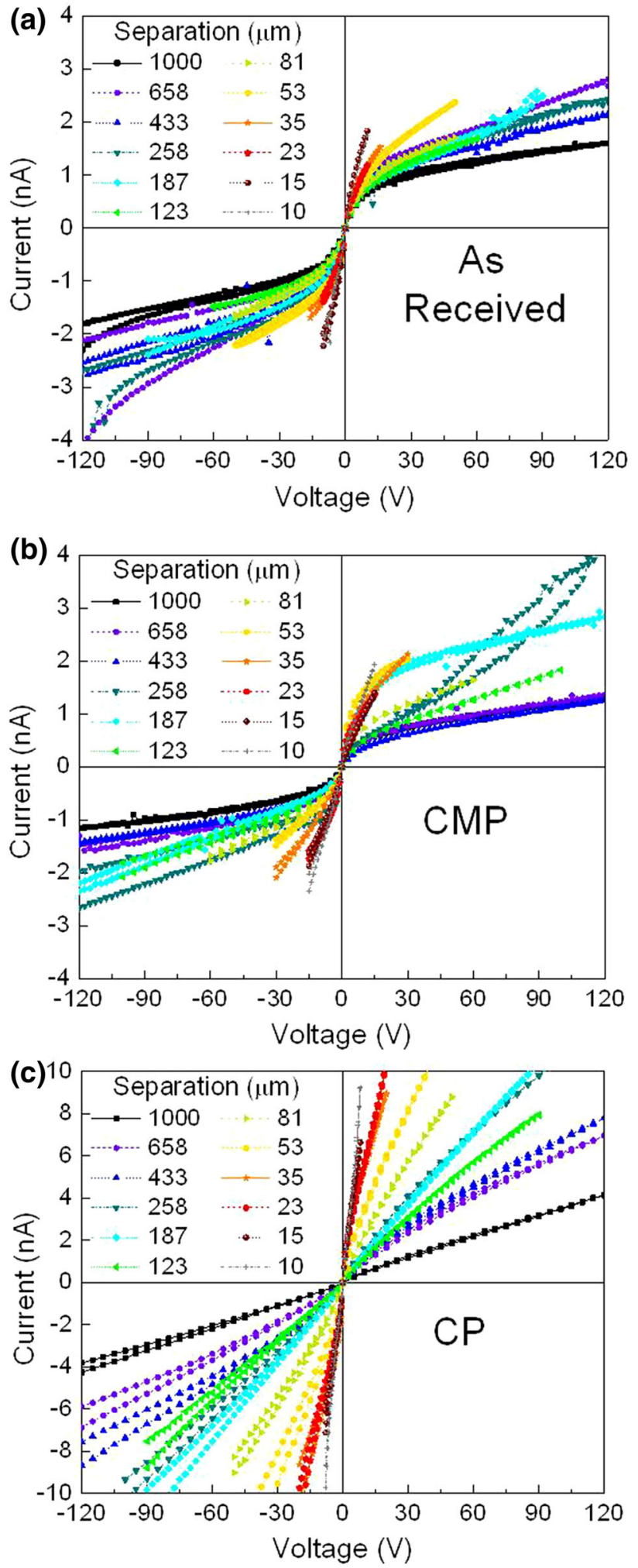

Fig. 7. $I-V$ curves for Au/CZT TLM contacts on (a) as received, (b) CMP-finished, and (c) CP-finished CZT surfaces. Different colors indicate increasing separation between the nearest contact lines, ranging from $10 \mu \mathrm{m}$ to $1000 \mu \mathrm{m}$. 
within a few hours of atmospheric exposure. A few nanometers of passivating oxide is likely to be insignificant compared with a damage layer several microns thick. The increase in leakage current caused by etching is probably because of removal of the damage layer, and so surface leakage may persist after etching, even if the surface is passivated. Charge trapping can alter the pulse shape from detection, so removal of the damage layer is important to carrier extraction.

\section{ACKNOWLEDGEMENTS}

The research at Sivananthan Laboratories and California State University at Northridge was supported by DTRA grant no. HDTRA1-10-1-0113. We thank the DTRA for their support.

\section{REFERENCES}

1. O. Limousine, Nucl. Instr. Method. A 504, 24 (2003).

2. C. Szeles, IEEE Trans. Nucl. Sci. 51, 1242 (2004).

3. G. Yang, W. Jie, Q. Li, T. Wang, G. Li, and H. Hua, J. Crystal Growth 283, 431 (2005).
4. Y.J. Li, G.L. Ma, X.N. Zhan, and W.Q. Jie, J. Electron. Mater. 31, 840 (2002)

5. Q. Li, W. Jie, Li Fu, X. Wang, and X. Zhang, Appl. Surf. Sci. 253, 1190 (2006).

6. E.J. Morton, M.A. Hosain, P. De Antonis, and A.M.D. Ede, Nucl. Instr. Method. A 458, 558 (2001).

7. S.H. Park, J.H. Ha, Y.H. Cho, H.S. Kim, S.M. Kang, Y.K. Kim, and J.K. Kim, IEEE Trans. Nucl. Sci. 55, 1547 (2008).

8. H. Chen, J. Tong, Z. Hu, D.T. Shi, G.H. Wu, K.-T. Chen, M.A. George, W.E. Collins, A. Burger, R.B. James, C.M. Stahle, and L.M. Bartlett, J. Appl. Phys. 80, 3509 (1996).

9. M.C. Duff, D.B. Hunter, A. Burger, M. Groza, V. Buliga, and D.R. Black, Appl. Surf. Sci. 254, 2889 (2008).

10. Q. Zheng, F. Dierre, M. Ayoub, J. Crocco, H. Bensalah, V. Corregidor, E. Alves, R. Fernandez-Ruiz, J.M. Pereze, and E. Dieguez, Cryst. Res. Technol. 46, 1131 (2011).

11. H. Chen, S.A. Awadalla, P. Marthandam, K. Iniewski, P.H. Lu, and G. Bindley, SPIE Proceedings: Hard X-Ray, Gamma-Ray, and Neutron Detector Physics XI 7449, 1 (2009).

12. A. Bensouici, V. Carcelen, J.L. Plaza, S. De Dios, N. Vijayan, J. Crocco, H. Bensalah, E. Dieguez, and M. Elaatmani, J. Crystal Growth 312, 2098 (2010).

13. F. Aqariden, S. Tari, K. Nissanka, Li Jin, N. Kioussis, R.E. Pimpinella, and M. Dobrowolska, J. Electron. Mater. 41, 2893 (2012). 